\title{
Avaliação de asfaltenos em petróleos para aplicação em gestão ambiental
}

Miranda, L. G. R. ${ }^{1^{*}}$; Rainha, K. P. ${ }^{2}$; Senna, J. N. ${ }^{1}$;Rodrigues, E. O. ${ }^{1}$; Amorim, J. V. ${ }^{3}$; Madeira, N. C. L. ${ }^{2}$;Ferreira, S. A. D. ${ }^{1}$; Castro, E. V. R. ${ }^{1}$

1 Departamento de Química, Universidade Federal do Espírito Santo, Vitória, ES, Brasil.

2 Programa de Pós-Graduação em Química, Universidade Federal do Espírito Santo, Vitória, ES, Brasil. 3 Departamento de Química, Universidade de Alberta, Edmonton, Alberta, Canadá.

*e-mail: luis.grmiranda@gmail.com

\section{RESUMO}

A maior parte da produção brasileira de petróleo é offshore. Isso implica em uma grande probabilidade de vazamentos de pequeno ou grande porte, que podem causar diversos prejuízos ao ambiente marinho. $O$ óleo exposto ao mar, ao longo do tempo sofre diversas transformações químicas, físicas e biológicas, sendo essas modificações denominadas intemperismo. Os principais processos de intemperismo são evaporação, dissolução, emulsificação, biodegradação, foto-oxidação, precipitação, adsorção, espalhamento e absorção pela biota. As modificações que ocorrem para cada petróleo são diferentes por dependerem da sua composição química, destacando os teores de asfaltenos. Neste trabalho, foram determinados os teores de asfaltenos e a validação do método para cinco amostras de diferentes correntes brasileiras em petróleo cru de acordo a ASTM D6560. Além disso, duas dessas amostras foram selecionadas para simulação do intemperismo por evaporação, a partir da destilação das mesmas a 150 $\stackrel{\circ}{ } \mathrm{C}, 200^{\circ} \mathrm{C}$ e $250^{\circ} \mathrm{C}$ e os teores de asfaltenos foram determinados para cada uma de acordo com a ASTM D3279. Foi observado a partir da simulação do intemperismo por evaporação que os teores de asfaltenos aumentam em relação ao tempo de exposição ao ambiente, devido a volatilização das frações mais leves, e essas variações podem implicar na precipitação e incrustação no leito marinho aumentando a toxicidade da água.

\begin{abstract}
Most of Brazil's oil production is offshore. This triggers to a high probability of both small and large leaks, which may cause several damages to the marine environment. When oil is exposed to the sea, it suffers several chemical, physical and biological modifications, which are called weathering. The main weathering processes are evaporation, dissolution, emulsification, biodegradation, photooxidation, precipitation, adsorption, scattering and absorption by biota. Modifications are different in each oil since it depends on its chemical composition, most importantly the asphaltenes content. For this research, asphaltenes content and method validation have been quantified for five samples of different Brazilian crude oil streams according to ASTM D6560. In addition, two of these samples have been selected to simulate evaporative weathering by distilling them at $150{ }^{\circ} \mathrm{C}, 200{ }^{\circ} \mathrm{C}$ and $250{ }^{\circ} \mathrm{C}$ and the asphaltenes contents have been quantified for each one of them, according to ASTM D3279. From the evaporation weathering simulation, it has been perceived that the asphaltenes contents increase in relation to the time of exposure to the environment due to the volatilization of the lightest fractions, and that these variations may cause precipitation and scale in the seabed, thus increasing the water toxicity.
\end{abstract}

Keywords: crude oil,asphaltene, weathering oil.

\section{Introdução}

O petróleo é uma das misturas mais complexas e diversificadas existentes na natureza, constituindo-se em grande parte por hidrocarbonetos saturados, aromáticos, resinas e asfaltenos, além dos compostos contendo heteroátomos e metais [1-3]. O processo de produção de petróleo no Brasil é predominantemente offshore. Em 2018, em torno de $95 \%$ da produtividade nacional ocorreram em campos marítimos [4]. A intensa atividade da indústria petrolífera nestes ambientes aumenta a probabilidade da ocorrência de algum tipo de vazamento de pequeno porte ou derramamentos de volumes maiores $[2,3]$.

O meio ambiente submete o petróleo derramado a uma série de transformações físicas, químicas e biológicas, conhecidas como intemperismo, que alteram suas 
características físico-químicas, toxicidade e interação com o ambiente. A ação da natureza é compreendida como uma remediação natural para mitigar o dano causado pela mancha de óleo [2,3,5]. Dentre os principais processos de intemperismo de petróleo encontram-se a evaporação, dissolução, emulsificação, biodegradação, foto-oxidação, precipitação, adsorção, espalhamento e absorção pela biota. A evaporação é a principal responsável pelas mudanças no óleo durante a intemperização, produzindo o petróleo intemperizado $[5,6]$. A progressão da exposição eleva a viscosidade e densidade do óleo intemperizado, aumentando a probabilidade quanto a formação de emulsões água/óleo estáveis. Essas variações estão relacionadas com a composição da matriz em questão, em especial aos teores de asfaltenos [2,3].

Os asfaltenos são estruturas de alto peso molecular e elevada aromaticidade, apresentando solubilidade em hidrocarbonetos aromáticos e insolubilidade nos alifáticos [1,6]. Essa classe de compostos é constituída por anéis aromáticos, cadeias laterais alifáticas, nitrogênio, oxigênio e enxofre, bem como metais traços como níquel e vanádio [1]. A fração asfaltênica do petróleo é classificada em tipo arquipélago e continental. A estrutura molecular e composição química dos asfaltenos não são completamente elucidadas, em decorrência à complexidade [7]. Em relação à exposição ao ambiente marinho, ainda são necessários mais resultados para uma melhor compreensão do comportamento em função às transformações intempéricas, interações ambientais, destinação e suas consequências de curto, médio e longo prazo.

As empresas produtoras de petróleo realizam a gestão de riscos ambientais, viabilizando recursos para a redução ou minimização dos impactos acidentais. A produção em um poço de petróleo inicia-se após aprovação do plano de contingência pelo órgão ambiental, que é um planejamento das possíveis medidas a serem tomadas em situações de emergência [8]. Esse documento é elaborado com base nos resultados de caracterização e avaliação do óleo em questão, demonstrando a importância do conhecimento prévio sobre a interação do ambiente marinho com as propriedades físico-químicas do petróleo, em especial dos asfaltenos, para que as ações de mitigação sejam assertivas. Estas informações auxiliam na avaliação da extensão de potenciais impactos ambientais e na tomada de decisão em situações de emergência [4,8]. Neste trabalho, objetiva-se determinar o teor de asfaltenos em petróleos crus e intemperizados, com a finalidade de observar as alterações da composição destas frações durante a simulação do intemperismo por evaporação.

\section{Metodologia}

Neste estudo foram avaliadas cinco amostras de petróleo provenientes de diferentes correntes marítimas do literal brasileiro, representando a amplitude de 15,4 a 32,4 API (American Petroleum Institute). O conjunto original de petróleos apresentou a representatividade de óleo leve ( $\left.\geq 31^{\circ} \mathrm{API}\right)$, médio ( $\geq 22$ a $\left.<31 \stackrel{\circ}{\circ} \mathrm{API}\right)$ e pesado (<22 ํAㄱ) [1,4].

2.1. Determinação do teor de asfalteno em petróleo cru A amostra homogeneizada foi pesada em balão de fundo chato $(250-500 \mathrm{~mL})$, seguindo as instruções da ASTM D6560 [9], e a cada $1 \mathrm{~g}$ adicionou-se $30 \mathrm{~mL}$ de $n$-heptano $99 \%$. O sistema foi aquecido sobre fluxo por $1 \mathrm{~h}$ e, posteriormente, deixado em repouso em câmara escura. A mistura foi filtrada à vácuo, recolhendo o sólido e refluxando o sistema com $100 \mathrm{~mL}$ de $n$ heptano por $1 \mathrm{~h}$. Depois o balão foi substituído por outro contendo $50 \mathrm{~mL}$ de tolueno PA, que também foi aquecido, sob as mesmas condições. Após essa etapa, o conteúdo foi rotaevaporado e o balão foi levado ao peso constante, utilizando balança analítica.

\subsection{Avaliação dos dados da ASTM D6560}

Os seguintes parâmetros estatísticos foram calculados utilizando os softwares Minitab® 14 e Microsoft Excel®: média $(\mu)$, desvio-padrão $(\sigma)$, variância $\left(\sigma^{2}\right)$, coeficiente de variação $(\mathrm{CV})$, repetibilidade $(r)$ e precisão intermediária $(\mathrm{R})$, que são representados pelas equações 1 à 6 , respectivamente. Neste trabalho, a precisão intermediária foi definida como variabilidade intralaboratorial das respostas amostrais, ao mesmo tempo, admitiu-se a equivalência quanto a reprodutibilidade (Equação 6). Finalmente, foram investigadas à similaridade entre os resultados pelo teste $\mathrm{F}$ (ANOVA) e t-Student, determinados para o nível de confiança de $95 \%[11,12]$.

$$
\begin{aligned}
& \mu=\sum \frac{\mathrm{x}}{\mathrm{n}} \\
& \sigma=\sqrt{\frac{\sum(x-\mu)^{2}}{n-1}} \\
& \sigma^{2}=\frac{\sum(x-\mu)^{2}}{n-1} \\
& \mathrm{CV}=\frac{\sigma}{\mu} \times 100 \\
& r=0,1 \times \mu \\
& \mathrm{R}=0,2 \times \mu
\end{aligned}
$$

2.3. Determinação dos teores de asfalteno em petróleo intemperizados

Aproximadamente $1 \mathrm{~g}$ da amostra homogeneizada foi pesada em erlenmeyer $250 \mathrm{~mL}$ e misturada a $100 \mathrm{~mL}$ de $n$-heptano 99\%, conforme a ASTM D3279 recomendada para os estudos com petróleos intemperizados [10].O conjunto foi submetido ao refluxo, aquecimento e agitação por $30 \mathrm{~min}$ e, posteriormente, deixado em repouso por $60 \mathrm{~min}$. Aqueceu-se a mistura até o intervalo de $38-49^{\circ} \mathrm{C}$, que 
logo após foi filtrada à vácuo em cadinho Gooch e funil com base de placa porosa. O sistema foi levado ao peso constante antes e após as etapas descritas acima.

\section{Discussão dos resultados}

Os resultados dos teores de asfaltenos obtidos para as cinco amostras de petróleo cru estão dispostos na Tabela 1. Evidencia-se também a diversidade quanto

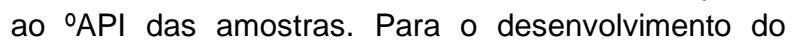
estudo estatístico, foram realizadas 4 réplicas. Dessa forma, cada petróleo original foi analisado em duplicata por 4 vezes, assim totalizando 8 determinações por amostra.

Tabela 1: Resultados das réplicas para a determinação dos teores de asfaltenos $(\% \mathrm{~m} / \mathrm{m})$ nos cinco petróleos com ${ }^{\circ} \mathrm{API}$.

\begin{tabular}{|c|c|c|c|c|c|c|c|c|c|}
\hline \multicolumn{10}{|c|}{ Amostras de petróleo cru } \\
\hline \multicolumn{2}{|c|}{ A } & \multicolumn{2}{|c|}{ B } & \multicolumn{2}{|c|}{ C } & \multicolumn{2}{|c|}{ D } & \multicolumn{2}{|c|}{ E } \\
\hline \multicolumn{2}{|c|}{ Duplicata } & \multicolumn{2}{|c|}{ Duplicata } & \multicolumn{2}{|c|}{ Duplicata } & \multicolumn{2}{|c|}{ Duplicata } & \multicolumn{2}{|c|}{ Duplicata } \\
\hline $1^{\mathrm{a}}$ & $2^{\mathrm{a}}$ & $1^{\mathrm{a}}$ & $2^{\mathrm{a}}$ & $1^{\underline{a}}$ & $2^{\mathrm{a}}$ & $1^{\mathrm{a}}$ & $2^{\mathrm{a}}$ & $1^{\mathrm{a}}$ & $2^{\mathrm{a}}$ \\
\hline 6,63 & 6,22 & 1,81 & 1,94 & 3,26 & 3,40 & 0,83 & 0,94 & 0,53 & 0,64 \\
\hline 6,35 & 6,67 & 2,00 & 1,99 & 3,37 & 3,30 & 0,88 & 0,87 & 0,59 & 0,52 \\
\hline 6,50 & 6,19 & 2,04 & 1,83 & 3,63 & 3,31 & 0,77 & 0,71 & 0,74 & 0,68 \\
\hline 6,43 & 6,69 & 1,97 & 2,07 & 3,13 & 3,44 & 0,99 & 0,78 & 0,77 & 0,88 \\
\hline \multicolumn{10}{|c|}{ @API dos petróleos } \\
\hline \multicolumn{2}{|c|}{15,4} & \multicolumn{2}{|c|}{17,1} & \multicolumn{2}{|c|}{20,0} & \multicolumn{2}{|c|}{32,4} & \multicolumn{2}{|c|}{29,5} \\
\hline
\end{tabular}

Para verificar a confiabilidade dos dados adquiridos, foram realizadas análises estatísticas descritivas e inferenciais. Os cálculos demonstraram que não houve discrepância nos resultados, considerando-se com inferências positivas. Ao analisar os valores obtidos pelo o teste $F$ e teste $t-S t u d e n t$, verificou-se que $F_{c a l} e$ $t_{\text {cal }}$ foram inferiores ao $F_{\text {tab }}$ e $t_{\text {tab }}($ Tabela 2) $[11,12]$.

Tabela 2: Estatística descritiva e inferencial aplicada aos dados obtidos a partir da ASTM D6560.

\begin{tabular}{|c|c|c|c|c|c|}
\hline & A & B & C & D & $E$ \\
\hline$\mu$ & 6,46 & 1,96 & 3,36 & 0,85 & 0,67 \\
\hline$\Sigma$ & 0,197 & 0,093 & 0,146 & 0,093 & 0,124 \\
\hline$\sigma^{2}$ & 0,039 & 0,009 & 0,021 & 0,009 & 0,015 \\
\hline CV & 3,05 & 4,76 & 4,36 & 10,90 & 18,60 \\
\hline $\mathrm{R}$ & 0,646 & 0,196 & 0,336 & 0,085 & 0,067 \\
\hline $\mathrm{R}$ & 1,29 & 0,391 & 0,671 & 0,169 & 0,014 \\
\hline$F_{\text {tab }}$ & & & 5,9847 & & \\
\hline $\mathrm{F}_{\text {cal }}$ & 0,055 & 0,001 & 0,018 & 0,383 & 0,056 \\
\hline $\mathrm{T}_{\mathrm{tab}}$ & & & 1,9432 & & \\
\hline $\mathrm{T}_{\text {cal }}$ & 0,2340 & 0,0351 & 0,1344 & 0,6190 & 0,2377 \\
\hline
\end{tabular}

A ferramenta estatística t-Student também é utilizada para expressar intervalos de confiança e comparar os resultados de um experimento, visto que a partir de um número limitado de medições não é possível encontrar a verdadeira média populacional, bem como o verdadeiro desvio-padrão. Os intervalos de confiança aderiram o nível de 95\% como representado na Figura 1, uma vez que quanto menor sua amplitude, maior a precisão da medida $[11,12]$. Desse modo, verificou-se que os óleos crus com os teores de asfaltenos mais extremos (A, D e E) evidenciaram uma menor precisão. No geral, os intervalos de confiança foram pequenos e admissíveis de acordo com a ASTM D6560.

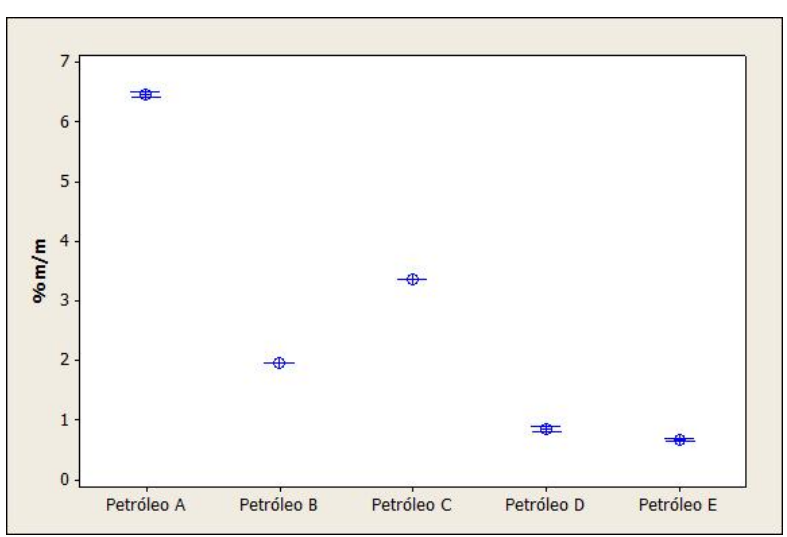

Figura 1: Intervalos de confiança referentes aos petróleos crus.

Para a investigação do comportamento dos asfaltenos submetidos à ação do intemperismo por evaporação, etapa simulada em laboratório, os petróleos originais foram destilados até o sistema atingir $150^{\circ} \mathrm{C}, 200^{\circ} \mathrm{C}$ e $250^{\circ} \mathrm{C}$. Cada temperatura correspondeu a um tempo de exposição no mar, com variação de 1 hora (Óleo-1H), 1 dia (Óleo-1D) e 1 semana (Óleo-1S), respectivamente. O petróleo intemperizado foi definido como o produto desta simulação [5,13,14].

Para execução da segunda etapa do presente trabalho, selecionaram-se apenas duas amostras de petróleo cru do conjunto inicial, que foram $\mathrm{B}\left(17,1^{\circ} \mathrm{API}\right)$ e C (20 -API). Os critérios determinantes para a consecução dessa escolha foram: 1) Disponibilidade de volume amostral que poderia ser destinada ao estudo; 2) Amostras com concentrações de asfaltenos acima de $1 \% \mathrm{~m} / \mathrm{m}$, uma vez que o teor acima de $3 \% \mathrm{~m} / \mathrm{m}$ é definido como crítico. A partir desse valor, o óleo tende a formar emulsão estável com a água o que dificulta e eleva o custo do processo de mitigação. Este índice também está relacionado com a capacidade desses compostos em precipitar $[6,7,13]$.

Posteriormente, os petróleos B e C cru se suas frações intemperizadas foram analisadas pelo método padrão ASTM D3279 (Tabela 3). A dificuldade quanto ao tempo demasiado para a obtenção das respostas e quantidade restrita dos materiais, inviabilizou a realização de réplicas. Logo, impossibilitou o desenvolvimento de um estudo estatístico mais minucioso, como o mostrado anteriormente. Portanto, nesta etapa os resultados provenientes da ASTM D3279 foram admitidos como adequados 
analiticamente através da comparação com a ASTM D6560, para as amostras de petróleo cru. A similaridade foi observada como segue na Tabela 2 (Boriginal com 1,96\% m/m e C-original com 3,36\% m/m) e Tabela 3 (B-original com 1,96\% $\mathrm{m} / \mathrm{m}$ e C-original com $3,30 \% \mathrm{~m} / \mathrm{m}$ ), de modo que os resultados não apresentaram diferença significativa. As respostas da ASTM D3279 foram avaliadas quanto à veracidade e conformidade metodológica, pois além da verificação dos resultados semelhantes mensurados no petróleo original por ambos, observou-se o contínuo atendimento aos critérios de avaliação do próprio método, em especial repetibilidade e reprodutibilidade [10].

Tabela 3: Teores de asfaltenos para os petróleos B e C originais e intemperizados de acordo com a ASTM D3279.

\begin{tabular}{ccccc}
\hline & B original & B-1H & B-1D & B1-1S \\
\hline$\% \mathbf{m} / \mathbf{m}$ & 1,96 & 2,39 & 2,45 & 2,59 \\
& & & & \\
\hline & C original & C-1H & C-1D & C-1S \\
\hline$\% \mathbf{m} / \mathbf{m}$ & 3,30 & 3,92 & 4,50 & 4,54 \\
\hline
\end{tabular}

Os teores de asfaltenos nestas amostras demonstraram um incremento linear na concentração $(\% \mathrm{~m} / \mathrm{m})$ dessas moléculas em função ao tempo de exposição do petróleo no mar e o intemperismo por evaporação, considerando a simulação. O processo de evaporação diminuiu a massa total do petróleo ao volatilizar as frações leves (baixa temperatura de ebulição) e, consequentemente, aumentando o teor de compostos mais pesados nos óleos intemperizados como, por exemplo, os asfaltenos [3,14]. O intemperismo por evaporação, geralmente, remove grande parte dos hidrocarbonetos de baixo peso molecular, enquanto realiza a degradação e favorece a biodegradação [2]. Após a simulação de uma semana de exposição, o aumento no teor de asfaltenos nas amostras B e C foi de $37,6 \%$ e $32,1 \%$, respectivamente, evidenciando a importância das ações primárias para a retirada do óleo exposto. Em situações de emergência, uma resposta rápida pode evitar a formação de emulsões ou agregação asfaltênica, pois o aumento da concentração dessa classe de compostos promove o surgimento de um filme viscoelástico de alta resistência em torno de gotículas de água em óleo, favorecendo a emulsificação [14]. A formação de emulsões estáveis atrapalha aplicação das medidas de contingência, além de diminuir as opções de resposta [13].

Os planos de contingência devem considerar a relação estabelecida entre a progressão do processo intempérico e o aumento da concentração de asfaltenos no ambiente. Nesse caso, as respostas de mitigação mais recomendação envolvem a abordagem por queima in situ e adição de produtos químicos para desestabilizar a interface entre água e óleo [2]. Os asfaltenos são associados aos problemas quanto à deposição no relevo oceânico, pois as moléculas agregadas são mais resistentes à degradação por microrganismos, dificultando a mitigação através da biorremediação $[3,7]$.

As propriedades físico-químicas em conjunto com a elucidação da composição do óleo auxiliam na calibração de modelos matemáticos capazes de predizer a evolução do intemperismo na mancha de óleo no mar [13,14]. A caracterização do petróleo fornece respaldo teórico para a elaboração dos planos de contingência pela gestão ambiental de riscos. Nesse contexto, aponta-se a escassez de estudos nesta área e, adicionalmente, que os asfaltenos podem impactar o ambiente marinho através dos processos de agregação, incrustação, precipitação, além de serem confundidos à alguma fonte de alimento pela fauna.

\section{Conclusão}

A determinação pela ASTM D6560 é eficiente de acordo com os resultados das análises estatísticas descritivas e inferenciais, com teor de asfaltenos variando de $0,67 \%$ a $6,46 \% \mathrm{~m} / \mathrm{m}$ para os cinco petróleos brasileiros. Os teores de asfaltenos são incrementados ao longo do tempo de exposição no meio ambiente, de acordo com a simulação do intemperismo por evaporação e pela aferição da concentração dessa classe de compostos por meio da ASTM D3279 nas amostras originais e intemperizadas. Os experimentos demonstram que quanto maior o tempo que o petróleo estiver exposto no ambiente marinho, maior será a concentração de asfaltenos, tendendo à precipitação e incrustação no leito marinho. As avaliações das ações intempéricas auxiliam a gerência de contingência, em casos de acidentes ambientais, evitando a contaminação e o aumento da toxicidade da água. É importante avaliar se o teor de asfaltenos ultrapassa $3 \%$, estimando também o tempo necessário para a ocorrência do aumento dessa concentração. Diante do exposto, estudos dessa natureza oferecem respaldo científico, otimizando o tempo e recursos nos processos de contingência em acidentes ambientais offshore.

\section{Agradecimentos}

Os autores agradecem ao Núcleo de Competências em Química do Petróleo, à Universidade Federal do Espírito Santo, à Petróleo Brasileiro S.A. e ao Centro de Pesquisa e Desenvolvimento Leopoldo Américo Miguez de Mello pelas instalações e apoio. 


\section{Referências}

[1] SPEIGHT, J. G. The Chemistry and Technology of Petroleum. Boca Raton/London/New York: CRC Press, 2007.

[2] TARR, M.A; ZITO, P.; OVERTON, E.B.; OLSON, G.M.; ADHIKARI, P.L.; REDDY, C.M. Oceanography. v. 29, p. 126-135, 2016.

[3] OVERTON, E.B.; WADE, T.L.; RANDOVÍC J.R.; MEYER, B.M.; MILES, M.S.; LARTER, S.R. Oceanography. v. 29, p. 50-63, 2016.

[4] Boletim de exploração e produção de petróleo e gás natural. Brasília. Disponível em: <https://bit.ly/2YiDAB0>. Acesso em: 03 Jun. 2019.

[5] DALING, P.S.;LEIRVIK, F.;ALMAS, T.K.;BRANDVIK, P.J.;HANSEN, B. H.;LEWIS, A.;REED, M. Marine Pollution Bulletin, v. 87, p. 300310, 2014.

[6] FINGAS, M. F.; FIELDHOUSE, B.; BOBRA, M. Marine Spill Response Corporations, v. 1, p. 237-242, 1993.

[7] DURAND, E.; CLEMANCEY, M.; LANCELIN, J. M.; VERSTRAETE, J.;ESPINAT, D.; QUOINEAUD, A. Energy and Fuels, v. 24, p. 1051-1062, 2010.
[8] Gestão de Riscos Ambientais - uma Comparação do Dimensionamento da Estrutura de Resposta a Vazamentos de Óleo no Brasil com as Diretrizes da Indústria do Petróleo. Rio de Janeiro. Disponível em: <https://bit.ly/31iaPkV>. Acesso em: 01 Ago. 2019.

[9] ASTM D6560. West Conshohocken: American Society for Testing and Materials: 2012.

[10] ASTM D3279. West Conshohocken: American Society for Testing and Materials: 2012.

[11] MINISTÉRIO DA AGRICULTURA PECUÁRIA E ABASTECIMENTO. Manual de Garantia da Qualidade Analítica. Brasília: MAPA/ACS: 2011.

[12] Instituto Nacional de Metrologia, Normalização e Qualidade Industrial. Orientações sobre validação de métodos de ensaios químicos, DOQ-CGCRE-008. Brasília. Disponível em: <https://bit.ly/2MG9UXx>. Acesso em: 02 Ago. 2019.

[13] DALING, P. S.; BRANDVIK, P. J.; MACKAY, D.; JOHANSEN, O. Oil \& Chemical Pollution, v. 7, p. 199224, 1990.

[14] FINGAS, M. Journal of petroleum science research, v. 2, p. 104-110, 2013. 